\title{
Gamma-secretase inhibitor does not modulate angiogenesis in colon adenocarcinoma in obese mice
}

\author{
Khazaei $\mathrm{M}^{1}$, Kalantari E², Saeidi H², ShabaniKia N², Tahergorabi Z ${ }^{1}$, Rashidi B ${ }^{3}$, Dana N ${ }^{4}$, Javanmard $\mathrm{SH}^{4}$ \\ Department of Physiology, Mashhad University of Medical Sciences, Mashhad, Iran. khazaeim@mums.ac.ir
}

\begin{abstract}
Background: Notch is a signaling molecule which plays a role in angiogenesis and $y$-secretase is required for processing of Notch. In this study, we investigated the effect of $\gamma$-secretase inhibitor (DAPT) on tumor angiogenesis in diet-induced obese mice.

Methods: 18 mice were divided into three groups; control, obese (diet-induced) and obese+DAPT. After 15 weeks, the obese mice were subjected for tumor induction of CT26 colon adenocarcinoma cells $\left(5 \times 10^{5}\right.$ cells). When the tumor size reached approximately $350 \pm 50 \mathrm{~mm}^{3}$, half of the obese animals received DAPT (10mg/kg/day) subcutaneously. Blood samples were taken after 14 days and the tumors harvested for immunohistochemical staining and capillary density were reported as CD31 positive cells $/ \mathrm{mm}^{2}$.

Results: The obese animals had higher serum leptin and NO concentrations, while, serum VEGF and VEGFR-1 concentrations were not different compare to control group. Administration of DAPT in obese mice significantly reduced serum VEGFR-1 and leptin concentrations and increased serum NO level $(p<0.05)$. Capillary density in the tumors of obese animals was not different compare to control groups. DAPT administration could not alter capillary density in the tumors. Conclusion: Administration of DAPT in obese mice altered serum angiogenic factors, however, it could not modulate tumor angiogenesis in diet-induced obese mice (Fig. 4, Ref. 26). Text in PDF www.elis.sk.

Key words: $y$-secretase, obese, angiogenesis, adenocarcinoma.
\end{abstract}

\section{Introduction}

Notch signaling is involved during angiogenesis in both physiological and pathological conditions (1,2). First time, Ellisen et al reported the role of Notch signaling in growth of T-cell acute lymphoblastic leukemia (3). Tumor suppressive role of Notch has been documented in some studies, while, the role of Notch signaling in promotion of tumor aniogenesis has also been reported $(1,4)]$. $\gamma$-secretase cleaves several proteins involved during angiogenesis process including Notch and inhibitors of this enzymes blocks the cleavage of Notch intracellular domain and inhibits Notch signaling. In the mice lacking $\gamma$-secretase activity, development of blood vessels is abnormal and they are at risk of cerebral hemorrhage (5). Therefore, it is suggested that $\gamma$-secretase inhibitors could modulate tumor angiogenesis.

Angiogenesis, the growth of new capillaries from preexisting ones, is a regulated process which is modulated by numerous angiogenic and antiangiogenic factors. Vascular endothelial growth factor (VEGF) and their receptors (VEGFR-1 and VEGFR-2) are the known factors which are involved in physiological and pathologi-

${ }^{1}$ Department of Physiology, Faculty of Medicine, Mashhad University of Medical Sciences, Mashhad, Iran, ${ }^{2}$ Students research Center, Faculty of Medicine, Isfahan University of Medical Sciences, Isfahan, Iran, ${ }^{3}$ Department of Anatomy, Isfahan University of Medical Sciences, Isfahan, Iran, and ${ }^{4}$ Applied Physiology Research Center, Isfahan University of Medical Sciences, Isfahan, Iran

Address for correspondence: M. Khazaei, MD, Department of Physiology, Faculty of Medicine, Mashhad University of Medical Sciences, Azadi square, Mashhad, Iran. $\quad$ Phone: +985138009227, Fax: +985138009217

Acknowledgement: The authors thank the Vice Chancellor Research of Isfahan University of Medical Sciences for financial support. cal angiogenesis $(6,7)$. Moreover, nitric oxide (NO) which is synthesized by endothelial NO synthase (eNOS), is the main endothelium-derived relaxing factor, which has angiogenic properties (8).

Since obesity is associated with increases risk of cancer including colorectal carcinoma (9), in the present study, we used a functional $\gamma$-secretase inhibitor, DAPT ( $N$-[ $N$-(3,5-DiflurophenaacetylL-alanyl)]-S-phenylglycinet-Butyl Ester) $(10,11)$ to determine the role of DAPT on colon adenocarcinoma cell angiogenesis in diet-induced obese mice.

\section{Materials and methods}

Animals

Male Balb/C mice, 5 weeks of age, were used in this study. The mice were purchased from the Pasteur Institute of Iran. The animals were maintained in animal room, $2-3$ mice per cage, at $20-25^{\circ} \mathrm{C}$ room temperature with $12 \mathrm{~h}$ light/dark cycle. To induce diet-induced obese mice, 12 mice were fed with high-fat diet (HFD) containing $59 \%$ fat, $27 \%$ carbohydrate, $14 \%$ protein (laboratories BioServ, Cat \#F3282, USA). To verify the effect of HFD on induction of dietinduced obesity, we also used a control group $(n=6)$ who received standard diet (Pasteur Institute of Iran). The experimental procedures were in accordance with the guideline of the animal care and ethical committee at the Isfahan University of Medical Sciences.

\section{Induction of tumor}

After 15 weeks, the obese mice were subjected for tumor induction by dorsal subcutaneous inoculation of CT26 colon adenocarcinoma cells $\left(5 \times 10^{5}\right.$ cells in $500 \mu$ l of phosphate buffered saline using 21 gauge needle) (REF). The animals were monitored 


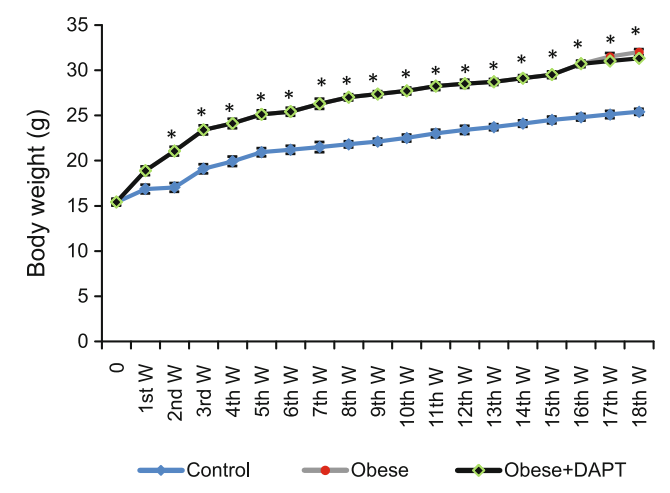

Fig. 1. Changes of body weight of the animals maintained on HFD and normal diet. * $p<0.05$ compared to normal diet group.

every day and when the tumor size reached approximately $350 \pm$ $50 \mathrm{~mm}^{3}$, the obese animals were randomly divided into two groups: obese and obese +DAPT. The DAPT was dissolved in DMSO (dimethyl sulfoxide) and injected $10 \mathrm{mg} / \mathrm{kg} /$ day, subcutaneously (10). The obese group received DMSO solution with the same volume. Blood samples were taken after 14 days and serums were separated and stored at $-70{ }^{\circ} \mathrm{C}$ for further analysis. Then, the animals were sacrificed and the tumors were harvested.

\section{Immunohistochemistry}

The tumors were fixed in $10 \%$ formalin solution. After paraffin embedding, the tissues were cut into 5 - $\mu \mathrm{m}$ sections, deparafinized and rehydrated. The sections were stained with standard immuno-
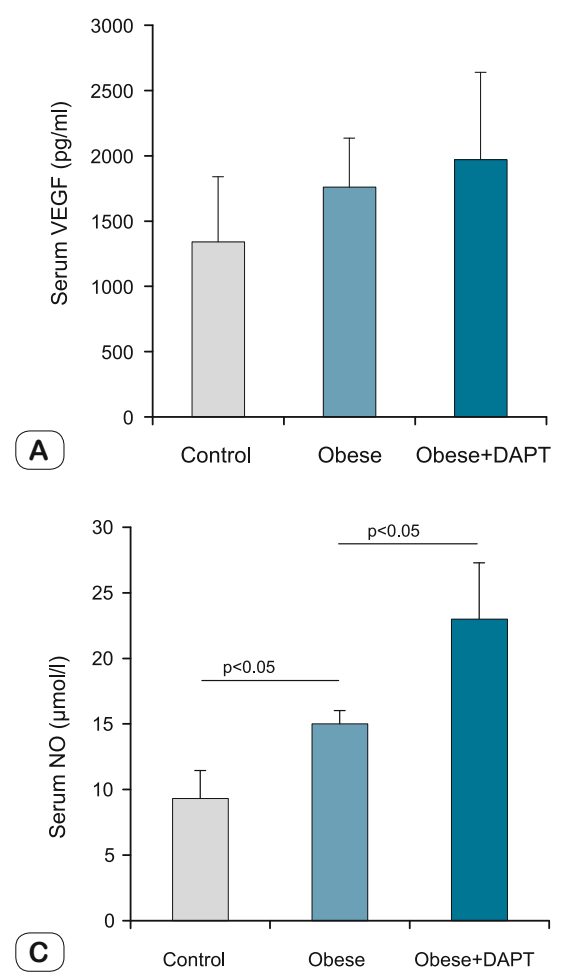

Fig. 2. Effect of DAPT on serum VEGF (A), VEGFR-1 (B), NO (C) and leptin (D) concentrations.

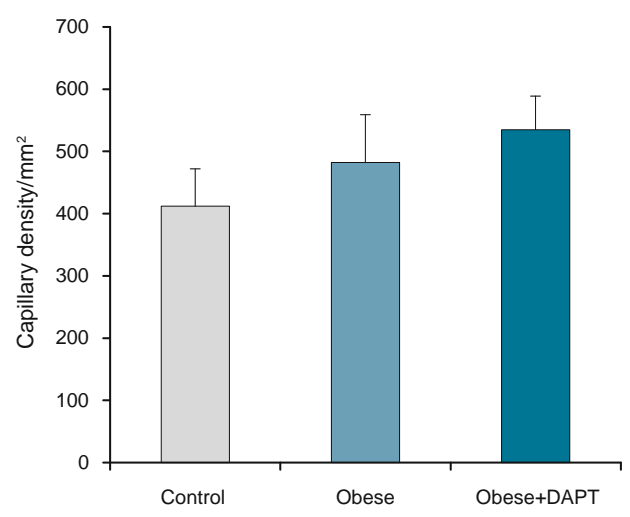

Fig. 3. Effect of DAPT on tumor capillary density (expressed as CD31 positive cells/mm2) in experimental groups.

histochemical protocol with a monoclonal rat anti-mouse CD31 antibody (Abcam Co.). Five sections were selected and CD31 positive cells were counted. Visual count was performed at $40 \mathrm{x}$ magnification in 20 fields of each section using an Olympus microscope and angiogenesis was reported as the number of CD31 positive cells per $\mathrm{mm}^{2}$.

\section{Serum angiogenic factor measurements}

Serum VEGF and VEGFR-1 were measured using enzymelinked immunosorbent assays (R\&D systems, Mineapolis, USA). Serum nitrite, the main metabolite of NO, was measured by Griess reagent method (Promega Corp, USA). Serum leptin levels were measured using specific sandwich enzyme immunoassay kit (In-
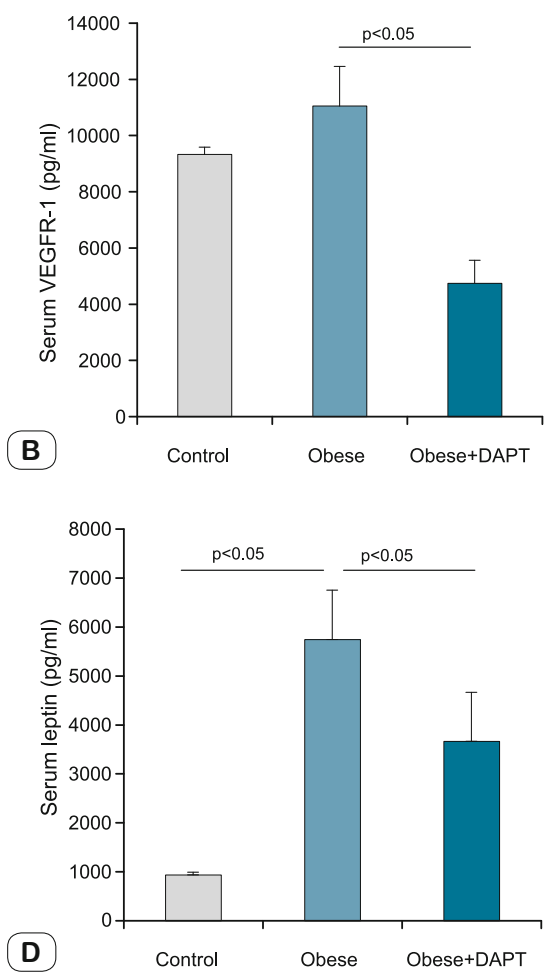
vitrogen, Camarillo, CA 93012) and measured according to the manufacturer's instructions.

\section{Statistical analysis}

The results are shown as the mean \pm SE. Statistical analysis of the data was performed using the One-Way ANOVA. $p<0.05$ was considered statistically significant.

\section{Results}

\section{Body weight}

As we expected, the obese mice presented body weights significantly higher than control group. Administration of DAPT could not significantly change body weight in the obese mice (Fig. 1).

\section{Serum measurements}

Figure 2 illustrates the changes of serum leptin, NO, VEGF and VEGFR-1 concentrations in experimental groups. The obese animals had higher serum leptin and NO concentrations, while, the serum VEGF and VEGFR-1 concentrations were not different compared to control group. Administration of DAPT significantly reduced serum VEGFR-1 and leptin concentrations in obese mice and increased serum NO level with no significant alteration in serum VEGF level.

\section{Capillary density in the tumor: effect of DAPT}

Capillary density expressed as CD31 positive cells $/ \mathrm{mm}^{2}$ in the tumors of obese animals was not different compared to control groups. DAPT administration could not significantly alter capillary density in the tumors (Fig. 3). Samples of immunohistochemical staining are shown in Figure 4.

\section{Discussion}

In the present study, obese animals had higher serum leptin and NO concentrations compared to control mice. Leptin is a protein with $16 \mathrm{kDa}$ which is secreted predominantly by adipocyte cells. Plasma level of leptin is proportional to adipose tissue mass and several studies reported this correlation. Higher NO in obese animals has been reported in previous studies. It is demonstrated that adipose tissue surrounding blood vessels is a source of NO overproduction in obese mice (12). Another study reported that HFD significantly increased hypothalamic NO production due to increased nNOS expression which may involve in insensitivity to leptin (13). NO is not only considered an anti-atherosclerotic factor (14), but is also involved in angiogenesis process (8).

In the present study, HFD increased serum nitrite level as a marker of NO production compared to control group. Besides the role of NO in food intake (15), it is involved in angiogenesis process, and has various antiatherosclerotic actions via inhibition of leukocyte adhesion and prevention of smooth muscle proliferation and protection. Abnormalities in the NO cyclic GMP pathway located at the subendothelial space could be involved in impaired vascular response and endothelial dysfunction in diabetic subjects (16). Benkhoff et al showed that HFD increased cerebral nNOS expression and cerebral and plasma nitrite levels in humans and mice (17).

After DAPT treatment for 14 days in obese mice, serum VEGFR-1 and leptin were decreased and serum NO increased compare to obese group not receiving DAPT. However, serum VEGF displayed no significant changes between DAPT-treated and untreated groups. VEGF is the known angiogenic factor which has a key role in physiological and pathological angiogenesis (6). VEGF has two receptors: VEGFR-1 and VEGFR-2 (18). VEGFR-1 is a potent negative regulator of VEGFR2 action (7). VEGFR1 is a negative regulator of angiogenesis, While, VEGFR2 has proangiogenic effect and stimulates endothelial cell proliferation and angiogenesis (19). In tumor tissue, VEGFR1 is the dominant receptor to VEGFR2 (20). Nitric oxide (NO) is the main endothelium-relaxing factor which is an angiogenic factor (8). Leptin is not only involved in food intake and energy balance, but also, it has angiogenic and mitogenic effects, and possess direct proangiogenic activity $(21,22)$. Therefore, it is possible that reduced serum VEGFR-1 and leptin and increased serum NO levels after DAPT treatment alters tumor angiogenesis in obese mice. In agreement with our results, a study in xenograft mouse model indicated that DAPT down-regulated VEGFR1 and up-regulated VEGFR2 (23). Interestingly, they found that there was no significant change in VEGF level between DAPT and DMSO groups.
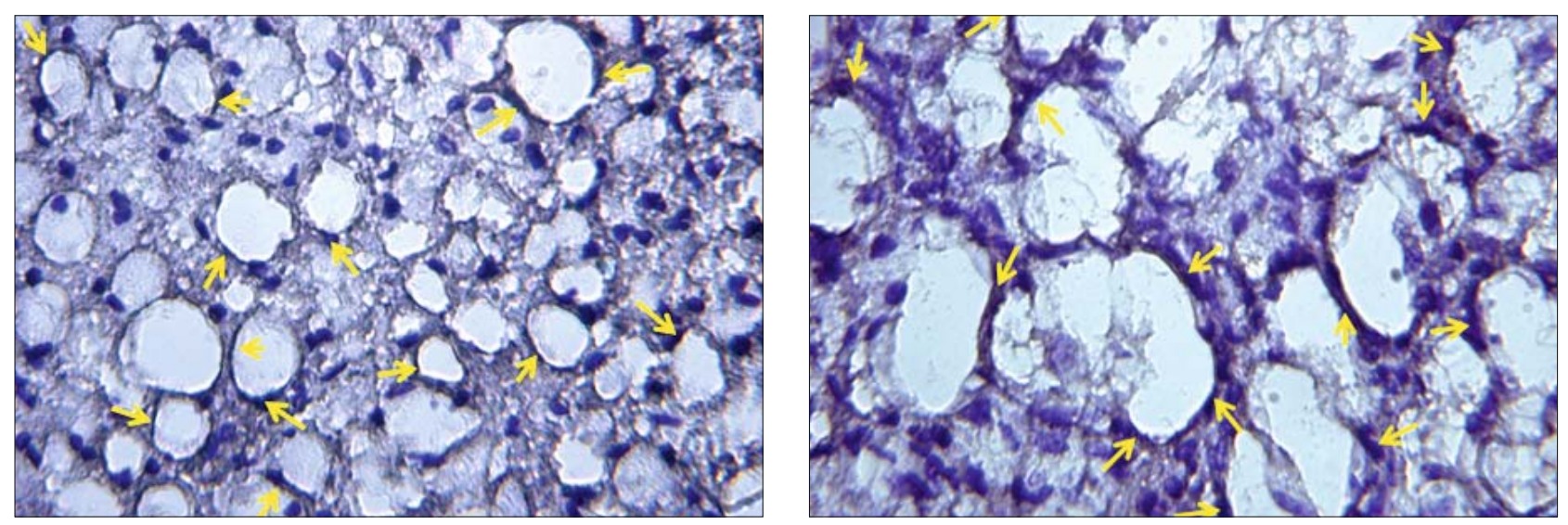

Fig. 4. Immunohistochemical staining of the tumors using anti CD31 antibody. Arrows indicate CD31 positive cells. 
$\gamma$-secretase processes several proteins which play a role during angiogenesis including Notch, CD44 or cadherin (1) and we expected that DAPT, a $\gamma$-secretase inhibitor, disrupts angiogenesis in tumor. Although studies indicated that $\gamma$-secretase inhibitors suppress tumor growth (23) and are involved in postnatal angiogenesis (24), however, in agreement with our results, recently, Zou $\mathrm{Y}$ et al reported that DAPT alone (without VEGF) had a little effect on cell proliferation and they concluded that although DAPT up-regulates VEGFR2 and down-regulates VEGFR1, however, it was not sufficient to affect endothelial cell proliferation (23). They also found that in an in vivo tumor model, DAPT disrupt functional vascular remodeling despite increasing the vessel number. In another study, DAPT failed to induce angiogenesis in a wound healing model (25). It is demonstrated that DAPT and DAPM (another $\gamma$-secretase inhibitor) dose-dependently inhibited angiogenesis, proliferation and differentiation of tumor endothelial cells (25). Furthermore, in hindlimb ischemia model, DAPT with VEGF promoted angiogenesis, however, intramuscular or intraperitoneal administration of DAPT alone, did not have the same level of improvement (26). Thus, it seems that the effect of DAPT on angiogenesis is dependent on the dose, model of angiogenesis and its administration with or without growth factors.

In conclusion, although administration of DAPT in obese mice with colon adenocarcinoma altered serum angiogenic factors, however, it could not change tumor angiogenesis. More studies need to clarify the exact role of $\gamma$-secretase inhibitors on angiogenesis process.

\section{References}

1. Rehman AO, Wang CY. Notch signaling in the regulation of tumor angiogenesis. Trends Cell Biol 2006; 16 (6): 293-300.

2. Mailhos C, Modlich U, Lewis J, Harris A, Bicknell R, Ish-Horowicz D. Delta4, an endothelial specific notch ligand expressed at sites of physiological and tumor angiogenesis. Differentiation 2001; 69 (2-3): 135-144.

3. Ellisen LW, Bird J, West DC, Soreng AL, Reynolds TC, Smith SD et al. TAN-1, the human homolog of the Drosophila notch gene, is broken by chromosomal translocations in T lymphoblastic neoplasms. Cell 1991; 66 (4): 649-661.

4. Leong KG, Karsan A. Recent insights into the role of Notch signaling in tumorigenesis. Blood 2006; 107 (6): 2223-2233.

5. Nakajima M, Yuasa S, Ueno M, Takakura N, Koseki H, Shirasawa T. Abnormal blood vessel development in mice lacking presenilin-1. Mech Dev 2003; 120 (6): 657-667.

6. Ferrara N. The role of VEGF in the regulation of physiological and pathological angiogenesis. EXS 2005; 94: 209-231.

7. Olsson AK, Dimberg A, Kreuger J, Claesson-Welsh L. VEGF receptor signalling - in control of vascular function. Nat Rev Mol Cell Biol 2006; 7 (5): 359-371.

8. Cooke JP, Losordo DW. Nitric oxide and angiogenesis. Circulation 2002; 105 (18): 2133-2135.

9. Calle EE, Kaaks R. Overweight, obesity and cancer: epidemiological evidence and proposed mechanisms. Nat Rev Cancer 2004; 4 (8): 579-591.

10. Paris D, Quadros A, Patel N, DelleDonne A, Humphrey J, Mullan $\mathbf{M}$. Inhibition of angiogenesis and tumor growth by beta and gammasecretase inhibitors. Eur J Pharmacol 2005; 514 (1): 1-15.
11. Dovey HF, John V, Anderson JP, Chen LZ, de Saint AP, Fang LY et al. Functional gamma-secretase inhibitors reduce beta-amyloid peptide levels in brain. J Neurochem 2001; 76 (1): 173-181.

12. Gil-Ortega M, Stucchi P, Guzman-Ruiz R, Cano V, Arribas S, Gonzalez MC et al. Adaptative nitric oxide overproduction in perivascular adipose tissue during early diet-induced obesity. Endocrinology 2010; 151 (7): 3299-3306.

13. Jang EH, Park CS, Lee SK, Pie JE, Kang JH. Excessive nitric oxide attenuates leptin-mediated signal transducer and activator of transcription 3 activation. Life Sci 2007; 80 (7): 609-617.

14. Wohlfart P, Xu H, Endlich A, Habermeier A, Closs EI, Hubschle T et al. Antiatherosclerotic effects of small-molecular-weight compounds enhancing endothelial nitric-oxide synthase (eNOS) expression and preventing eNOS uncoupling. J Pharmacol Exp Ther 2008; 325 (2): 370-379.

15. Jahng JW, Lee JY, Yoo SB, Kim YM, Ryu V, Kang DW et al. Refeeding-induced expression of neuronal nitric oxide synthase in the rat paraventricular nucleus. Brain Res 2005; 1048 (1-2): 185-192.

16. Maejima K, Nakano S, Himeno M, Tsuda S, Makiishi H, Ito T et al. Increased basal levels of plasma nitric oxide in Type 2 diabetic subjects. Relationship to microvascular complications. J Diabetes Complications 2001; 15 (3): 135-143.

17. Benkhoff S, Loot AE, Pierson I, Sturza A, Kohlstedt K, Fleming I et al. Leptin potentiates endothelium-dependent relaxation by inducing endothelial expression of neuronal NO synthase. Arterioscler Thromb Vasc Biol 2012; 32 (7): 1605-1612.

18. Rahimi N. Vascular endothelial growth factor receptors: molecular mechanisms of activation and therapeutic potentials. Exp Eye Res 2006; 83 (5): 1005-1016.

19. Wu FT, Stefanini MO, Mac GF, Kontos CD, Annex BH, Popel AS. A systems biology perspective on SVEGFR1: its biological function, pathogenic role and therapeutic use. J Cell Mol Med 2010; 14 (3): 528-552.

20. Zhang Z, Neiva KG, Lingen MW, Ellis LM, Nor JE. VEGF-dependent tumor angiogenesis requires inverse and reciprocal regulation of VEGFR1 and VEGFR2. Cell Death Differ 2010; 17 (3): 499-512.

21. Anagnostoulis S, Karayiannakis AJ, Lambropoulou M, Efthimiadou A, Polychronidis A, Simopoulos C. Human leptin induces angiogenesis in vivo. Cytokine 2008; 42 (3): 353-357.

22. Hoda MR, Popken G. Mitogenic and anti-apoptotic actions of adipocyte-derived hormone leptin in prostate cancer cells. BJU Int 2008; 102 (3): 383-388.

23. Zou Y, Cao Y, Yue Z, Liu J. Gamma-secretase inhibitor DAPT suppresses glioblastoma growth via uncoupling of tumor vessel density from vessel function. Clin Exp Med 2012.

24. Hayashi H, Nakagami H, Takami Y, Sato N, Saito Y, Nishikawa T et al. Involvement of gamma-secretase in postnatal angiogenesis. Biochem Biophys Res Commun 2007; 363 (3): 584-590.

25. Caiado F, Real C, Carvalho T, Dias S. Notch pathway modulation on bone marrow-derived vascular precursor cells regulates their angiogenic and wound healing potential. PLoS One 2008; 3 (11): e3752.

26. Cao L, Arany PR, Wang YS, Mooney DJ. Promoting angiogenesis via manipulation of VEGF responsiveness with notch signaling. Biomaterials 2009; 30 (25): 4085-4093.

Received September 7, 2013. Accepted October 1, 2013. 